\title{
Tratamento estético: Aumento de coroa clínica inferior
}

\author{
Aesthetic treatment: Increased lower clinical crown \\ Tratamiento estético: Aumento de la coroa clínica inferior
}

Recebido: 01/02/2021 | Revisado: 07/02/2021 | Aceito: 07/02/2021 | Publicado: 08/02/2021

Thalya Horsth Maltarollo

ORCID: https://orcid.org/0000-0002-6697-1905 Centro Universitário das Faculdades Metropolitanas Unidas, Brasil

E-mail: thalyamaltarollo@gmail.com

Melissa de Santana Lucio

ORCID: https://orcid.org/0000-0001-5919-6771 Centro Universitário das Faculdades Metropolitanas Unidas, Brasil

E-mail:me_santana@outlook.com

Rabbith Ive Carolina Shitsuka Risemberg ORCID: https://orcid.org/0000-0003-1640-8898 Universidade Cruzeiro do Sul, Brasil

E-mail: rabbith.ive@gmail.com

Irineu Gregnanin Pedron

ORCID: https://orcid.org/0000-0002-2677-5539

Universidade Brasil, Brasil

E-mail: igpedron@alumni.usp.br

Silvia Linard Mercelino Friggi

ORCID: https://orcid.org/0000-0003-0022-7662

Centro Universitário das Faculdades Metropolitanas Unidas, Brasil

E-mail: silvialinard@hotmail.com

\begin{abstract}
Resumo
Atualmente a busca por um sorriso perfeito vem aumentando e se tornando uma exigência comum na prática clínica odontológica. Este artigo tem como objetivo apresentar um relato de caso de um tratamento estético cirúrgico para um paciente com excesso de gengiva dos dentes inferiores. A cirurgia de aumento de coroa clínica demonstrou uma forma de devolver as distâncias biológicas adequadas para restabelecer saúde, função e estética do caso proposto, proporcionando resultados satisfatórios tanto para o paciente quanto para o cirurgião-dentista.
\end{abstract}

Palavras-chave: Estética; Periodontia; Sorriso.

\begin{abstract}
Currently the search for a perfect smile has been increasing and becoming a common requirement in clinical dental practice. This article has the objective to present a case report of a surgical aesthetic treatment for a patient with a excess gingiva of the lower teeth. Surgical crown augmentation surgery demonstrated a way to return the appropriate biological distances to restore health, function and aesthetics of the proposed case, providing satisfactory results for both the patient and the dentist.
\end{abstract}

Keywords: Aesthetics; Periodontics; Smile.

\section{Resumen}

Actualmente, la búsqueda de una sonrisa perfecta ha aumentado y se ha convertido en un requisito común en la práctica clínica dental. El propósito de este artículo es presentar un informe de caso de un tratamiento estético quirúrgico para un paciente con exceso de encía de los dientes inferiores. La cirugía quirúrgica de aumento de la corona demostró una forma de devolver las distancias biológicas apropiadas para restaurar la salud, la función y la estética del caso propuesto, proporcionando resultados satisfactorios tanto para el paciente como para el dentista.

Palabras clave: Estética; Periodoncia; Sonreír.

\section{Introdução}

A estética tem como significado beleza e aparência harmoniosa em suas formas (Dicionário Houaiss, 2019). Atualmente a busca pela estética vem crescendo, causando impacto no dia a dia da prática clínica Odontológica.

Para uma estética facial agradável é necessária a harmonia entre as formas constituintes da face e quando algo interfere nesta, como nos casos de exposição em excesso de gengiva ao sorrir e falar, ou sorriso gengival, pode gerar impacto negativo 
na qualidade de vida, no bem estar psicossocial e emocional do paciente (Lukez, et al, 2015). Indo ao encontro dessas necessidades, torna-se importante para os pacientes a busca pela estética facial agradável e bem aceita na sociedade.

Segundo Pithon, et al, (2014), de acordo com os padrões estéticos atuais, a exposição de uma pequena faixa de gengiva é necessária para a constituição do sorriso, quando em excesso prejudica a harmonia deste sendo, segundo a American Academy of Periodontoly, considerado como uma deformidade mucogengival em torno dos dentes (Armitage, 1999).

De acordo com Braga et al (2014), Flôres \& Tramontina, (1996), Lourenço et al, (2017), várias são as etiologias que levam ao sorriso gengival como a erupção passiva alterada (EPA), quando a gengiva não migra apicalmente durante o processo de erupção dental, presença de lábio superior curto, excesso maxilar anterior, extrusão dentoalveolar, hiperatividade do lábio superior, hiperplasia gengival e a combinação de vários fatores, e seu conhecimento é fundamental para obtenção do diagnóstico e decisão do tratamento a ser realizado necessitando, em muitos dos casos, ser corrigida através de procedimentos cirúrgicos.

A cirurgia de aumento de coroa clínica é uma das formas de tratamento escolhida para se obter o sucesso no restabelecimento do equilíbrio das estruturas dento-gengivais em casos de EPA, e a decisão pelo tipo da técnica cirúrgica pertinente deve ser guiado por um diagnóstico e planejamento adequados (Cistóvan et al,2019; Borges et al.,2012; Brilhante et al, 2014; Gonzales \& Almeida, 2011).

Esse estudo tem como objetivo descrever um relato de caso clínico de aumento de coroa clínica inferior usando a técnica de retalho de espessura total com excisão de margens associada à osteotomia e osteoplastia para tratamento de erupção passiva alterada do tipo $1 \mathrm{~B}$.

\section{Metodologia}

Um estudo de caso é um tipo de estudo centrado em um fenômeno e que busca de descrevê-lo de modo mais detalhado possível como afirma (Yin, 2015; Pereira et al., 2018). Na presente pesquisa realiza-se um estudo de caso clínico o qual contou com o termo de consentimento livre e esclarecido (TCLE). A pesquisa seguiu os preceitos éticos de acordo com a Declaração de Helsinque.

\section{Relato de Caso}

Paciente LFS, 35 anos, gênero masculino, leucoderma, sem alteração sistêmica, procurou a Clínica Integral do Adulto III, no Centro Universitário das Faculdades Metropolitanas Unidas (FMU), com a queixa principal de possuir “dentes curtos”, excesso de gengiva ao sorrir e que isto o atrapalhava em seu convívio social (Figura 1). 
Figura 1. Pré-operatório.

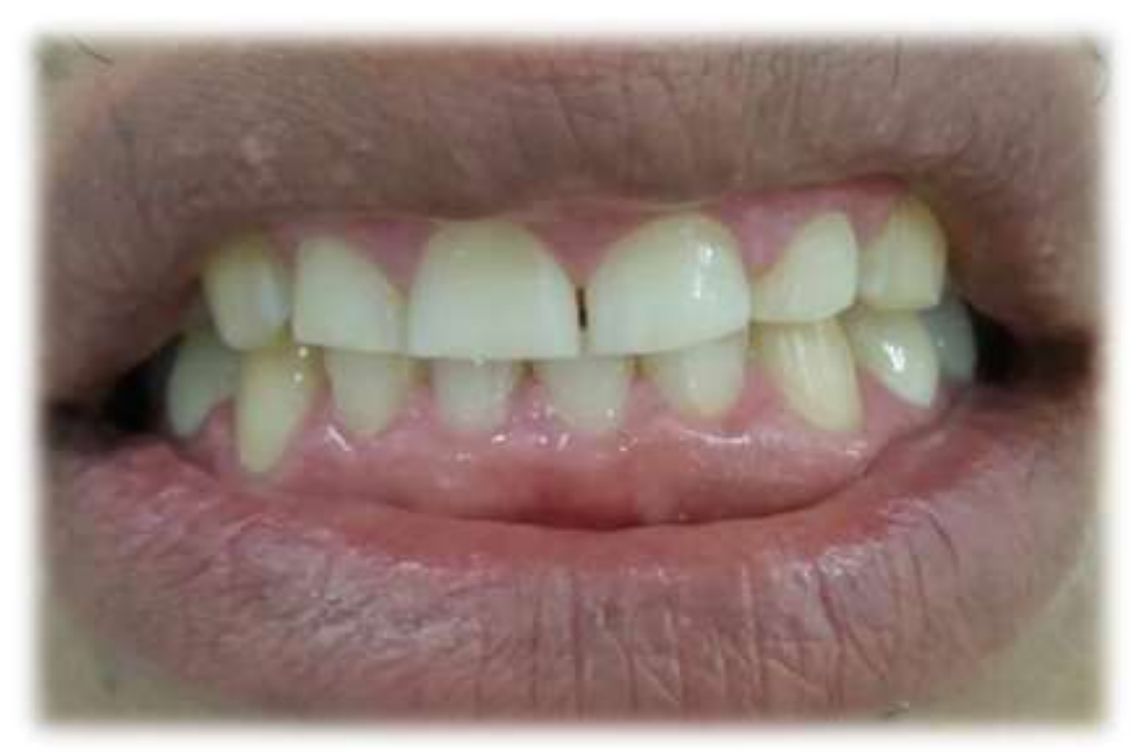

Fonte: Autores.

Observa-se os elementos dentais com pouca dimensão vertical e motivo da queixa. Inicialmente foi realizada a anamnese seguida de avaliação do paciente. Durante a avaliação clínica foi constatada a presença de dentes curtos, quadrados e excesso gengival dos dentes anteroinferiores.

Por meio do exame periodontal simplificado (PSR), realizado com a sonda OMS, ou sonda WHO (Hu Friedy $\left.{ }^{\circledR}\right)$, pôdese observar gengivite e ausência de bolsas periodontais. Como o paciente possuía alteração da localização da margem gengival em relação a junção esmalte-cemento (JEC) foi realizado o periograma, de acordo com Tekavec et al, 1993, do sextante E (dentes anteroinferiores), com a sonda Carolina do Norte (Hu Friedy $\left.{ }^{\circledR}\right)$, e radiografias periapicais para essa região e verificouse que o nível da crista óssea alveolar (COA) se encontrava no nível da JEC, diagnosticando-o como portador de erupção passiva alterada do tipo $1 \mathrm{~B}$.

O paciente foi submetido ao tratamento periodontal de orientação de higiene bucal, através da técnica de escovação de Bass modificada e do uso do fio dental como orientado em Lindhe (2018), e posterior raspagem supragengival de todos os dentes. No retorno para reavaliação, foi notado saúde periodontal e então procedeu-se para a cirurgia de aumento de coroa clínica.

Incialmente foi realizado a antissepsia intraoral e extraoral (digluconato de clorexidina $0,12 \%$ e $2 \%$, respectivamente) e técnica anestésica de bloqueio do nervo mentual bilateral e interpapilar do sextante E (mepivacaína 2\% 1: 1000.000). Foi feita a sondagem e marcação dos pontos sangrantes, com o auxílio de uma sonda milimetrada Carolina do Norte (Hu Friedy ${ }^{\circledR}$ ), na altura da JEC, para delimitar o tecido gengival em excesso. Em seguida, com o gengivótomo de Kirkland $\left(\right.$ Hu Friedy $\left.{ }^{\circledR}\right)$, foi feita uma incisão em bisel externo a $45^{\circ}$ abaixo da marcação, seguida pela incisão interproximal, com o gengivótomo de Orban (Hu Friedy ${ }^{\circledR}$ ), paralelo ao dente, com o objetivo de descolar a porção de gengiva interproximal e com o auxílio das curetas de Gracey 3/4 e 5/6 (Hu Friedy ${ }^{\circledR}$ ) foi removido o colarinho e tecido de granulação.

Na elevação do retalho de espessura total, foi utilizada uma lâmina de bisturi 15C em bisel interno e um descolador de Molt (Hu Friedy $\left.{ }^{\circledR}\right)$ para fazer a sindesmotomia permitindo o acesso e visualização do osso. Foi feita a osteoplastia e osteotomia, em alta rotação com a broca H 207 D (haste em alta rotação longa - KOMET ${ }^{\circledR}$ ) com refrigeração, restabelecendo a distância necessária para as estruturas teciduais supracrestais (espaço biológico). 
O retalho foi reposicionado coronalmente ao nível da JEC e suturado com fio de nylon 4.0 (Ethicon ${ }^{\circledR}$ ) utilizando a técnica colchoeiro vertical (Figura 2).

Figura 2. Pós-operatório imediato.

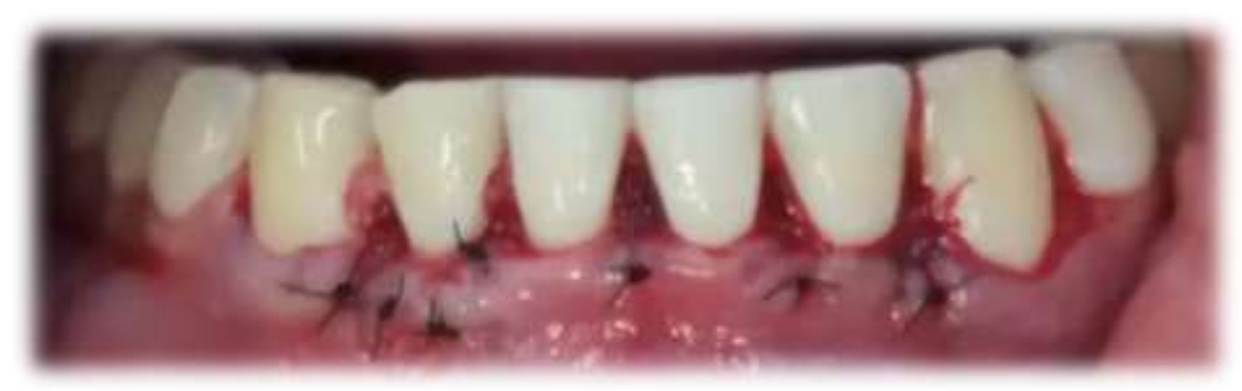

Fonte: Autores.

A figura apresenta os locais nos quais foram feitas cirurgias na região maxilar. Para os cuidados pós-operatórios foi prescrito anti-inflamatório (Ibuprofeno 600mg) a cada oito horas durante 4 dias e bochechos de $15 \mathrm{ml}$ de digluconato de clorexidina a $0,12 \%$ duas vezes ao dia após 24 horas do procedimento cirúrgico, durante 14 dias.

A Figura 3 apresenta a imagem do pós-operatório de 7 dias. Em 21 dias, decorridos de cirurgia, o paciente apresentava um estado de saúde periodontal satisfatório.

Figura 3. Pós-operatório 7 dias.

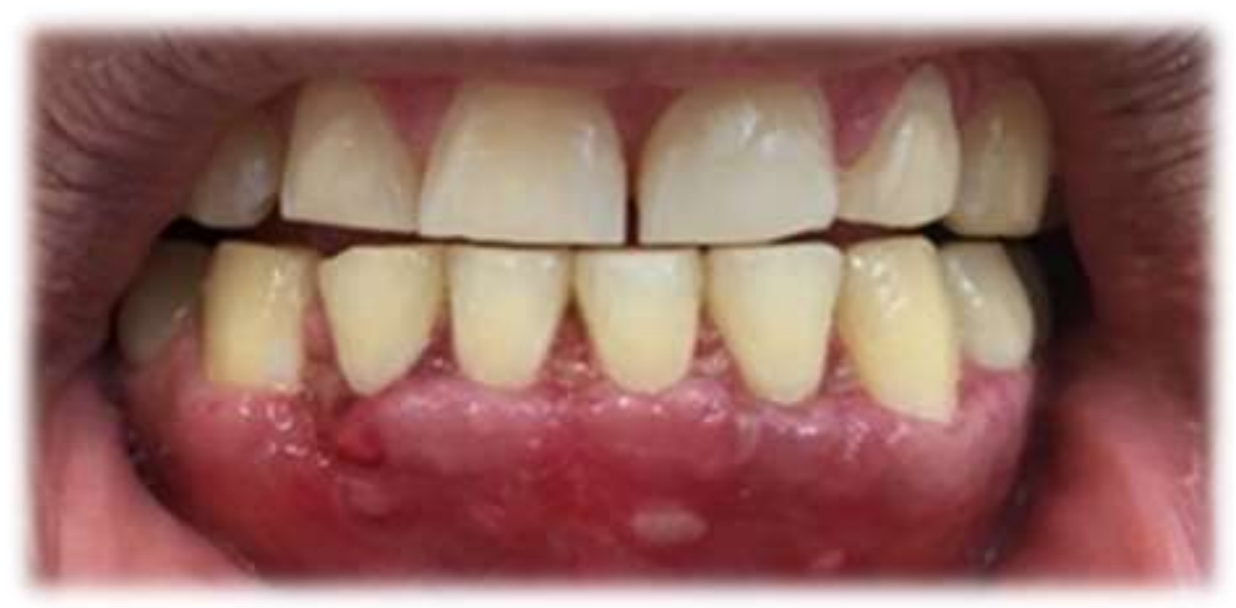

Fonte: Autores.

Por meio desta imagem, verifica-se que os pontos cirúrgicos foram removidos. A Figura 4 apresenta a imagem tirada após 14 dias. 
Research, Society and Development, v.10, n. 2, e26310212524, 2020

(CC BY 4.0) | ISSN 2525-3409 | DOI: http://dx.doi.org/10.33448/rsd-v10i2.12524

Figura 4. Pós-operatório 14 dias.

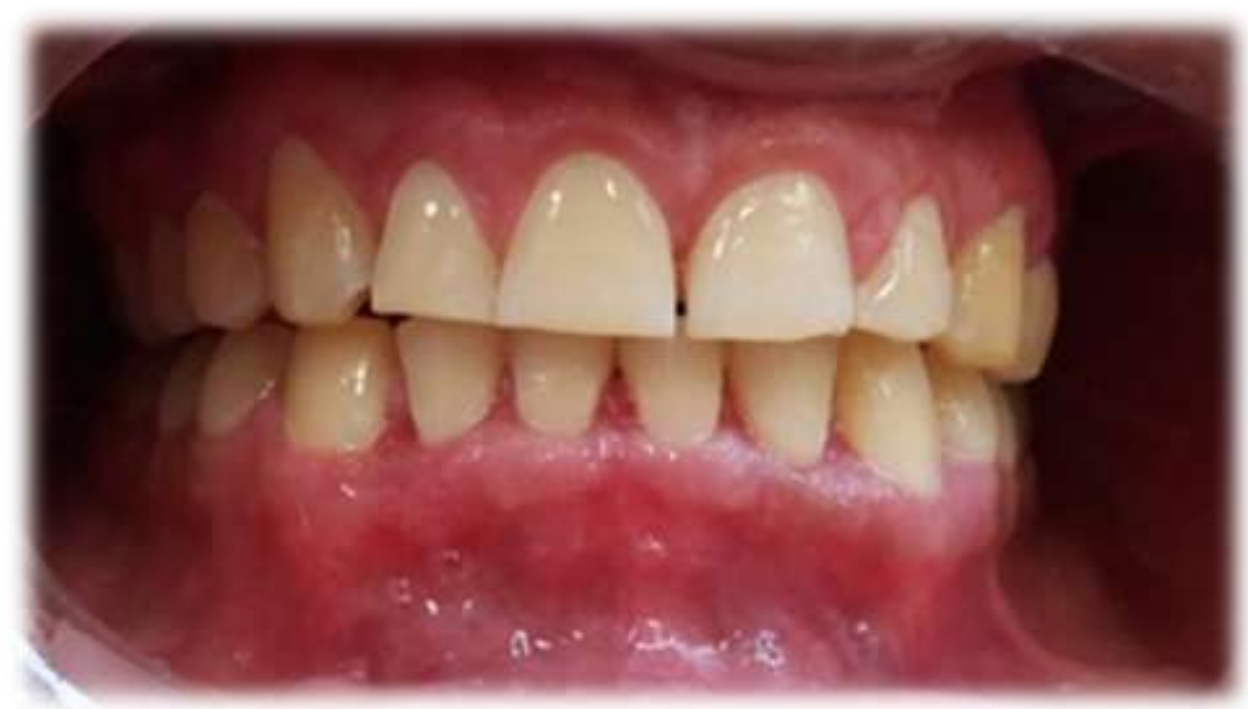

Fonte: Autores.

Verifica-se, por meio da figura, que nenhum evento adverso foi encontrado, a região gengival apresenta boa coloração e, tudo aponta para o êxito do trabalho realizado.

No retorno após seis meses (Figura 5), foi feito uma reavaliação periodontal constatando uma manutenção das distâncias estabelecidas na cirurgia.

Figura 5. Aspecto final do sorriso após seis meses.

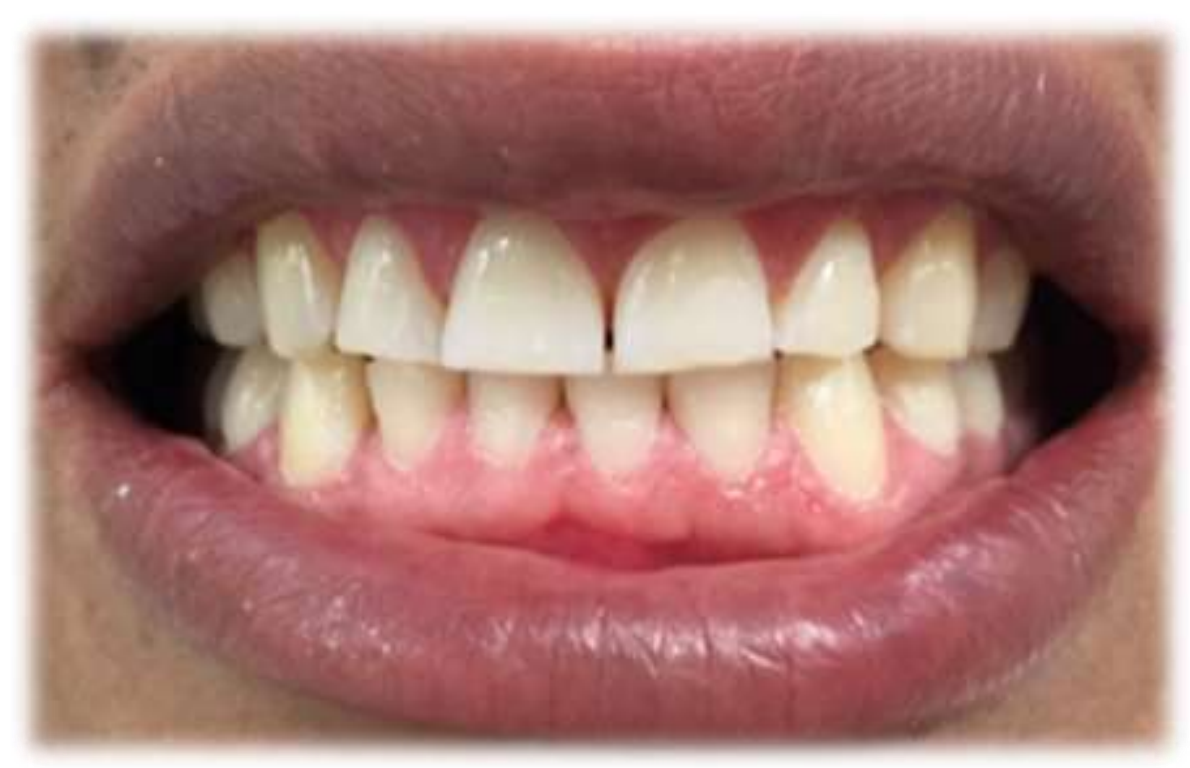

Fonte: Autores.

A figura permite observar que houve um ganho na altura vertical e que não foi excessivo mas permitiu uma melhora estética bucal na paciente. 


\section{Discussão}

As cirurgias plásticas periodontais são indicadas para alcançar a harmonia das estruturas dento-gengivais proporcionando, além da estética, função e saúde. Em pacientes que apresentam sorriso gengival proveniente da EPA, as técnicas que visam o aumento de coroa clínica são as mais indicadas e para o planejamento da correta técnica cirúrgica é imprescindível um diagnóstico onde as queixas e expectativas do paciente também sejam levadas em consideração (Gonzales \& Almeida, 2011; Bordin et al, 2010).

Conforme Mele et al, 2018, diagnóstico de EPA se baseia através de dados obtidos na anamnese, avaliação clínica extra oral da simetria facial e linha labial, na investigação clínica intrabucal da quantidade de gengiva queratinizada, o biotipo periodontal, a localização da junção esmalte-cemento (JEC) em relação à crista óssea alveolar (COA) por meio de sondagem transulcular (periograma) e análises radiográficas.

A sua classificação é baseada na localização da junção mucogengival (JMG) à COA e ao posicionamento da COA à JEC. Na EPA do tipo I a JMG é comumente apical à COA, no tipo II a gengiva inserida está situada na coroa dental com a JMG na altura da JEC. No subgrupo A, da EPA, a COA está a uma distância normal ( 1 a $2 \mathrm{~mm}$ apicalmente) da JEC, no subgrupo B a COA permanece no nível ou coronal da JEC (Gonzales \& Almeida, 2011; Mele et al, 2018).

Segundo Dantas et al (2012), Gonzales e Almeida (2011), as escolhas cirúrgicas para aumento de coroa clínica em dentes anteriores utilizadas comumente são: gengivectomia, cirurgia a retalho com osteotmia e/ou, osteoplastia e cirurgia sem elevação de retalho com osteotomia (Técnica de Flapless) e a sua escolha varia de acordo com a classificação do tipo da EPA, podendo ser: Tipo IA a Gengivectomia ou Retalho de bisel interno; Tipo IB o Retalho de bisel interno com osteotomia; Tipo IIA o Retalho com posicionamento apical; Tipo IIB o Retalho com posicionamento apical com osteotomia.

Os exames radiográficos também são de grande valor no planejamento da técnica a ser utilizada, uma vez que eles nos dão uma mensuração dos parâmetros clínicos das distâncias biológicas, como a radiografia periapical e a Tomografia Computadorizada de Feixe Cônico (TCFC), sendo esta última também capaz de evidenciar o biotipo periodontal (Kahn et al, 2014).

Diante das condições financeiras do paciente, não foi possível a realização de uma TCFC, e por isso o biotipo ósseo do paciente só foi comprovado como espesso após a elevação do retalho de espessura total havendo, assim, a necessidade da realização da osteoplastia para remover o osso também em espessura (Lindhe \& Lang, 2018).

Quando o nível da crista óssea se encontra próximo ou no nível da JEC, há a necessidade de uma remodelação óssea para restabelecer as distâncias biológicas no nível requerido (Alvarenga et al. 2018). A distância do espaço biológico, foi estabelecida por Gargiulo et al. 1961 em torno de 3,0 mm, e pode variar entre as pessoas, estando presente em todas as dentições saudáveis (Bordin et al. 2010).

O termo gengivectomia costuma ser erroneamente utilizado em casos onde se relata um caso de aumento de coroa clínica com retalho, pois são técnicas distintas e ambas têm indicações específicas. Enquanto a gengivectomia serve para aumento de coroa para eliminação de bolsas periodontais, não tendo como objetivo o acesso ao osso, a cirurgia a retalho é indicada para casos onde há um excesso de tecido ósseo, necessitando ser acessado e removido (Lindhe \& Lang, 2018).

Uma outra opção cirúrgica para aumento de coroa clínica estética é a técnica sem elevação de retalho "Flapless", indicada em casos de biotipo periodontal intermediário e delgado, onde reduz o tempo de cicatrização tecidual, inflamação local e, devido a isto, gera melhor conforto pós-operatório (Lemes et al 2018).

Existem tratamentos não cirúrgicos para casos de excesso gengival, como a toxina botulínica, que em comparação aos procedimentos cirúrgicos mais invasivos, é uma alternativa mais conservadora, rápida, segura, eficaz, que produz resultados harmônicos e agradáveis, indicado principalmente para casos de excesso gengival em maxila, interferindo na contração dos 
músculos elevadores do lábio quando respeitada a dose apropriada e o tipo de sorriso. Sua duração é relativamente curta, precisando ser refeita a cada 6 meses para obter sucesso e satisfação do paciente (Pedron, 2014).

O caso exposto nesse artigo é incomum na literatura trata-se de excesso gengival da região inferior, na mandíbula, e pode não aparecer, em muitos casos durante o sorriso, mas durante a gesticulação das palavras e expressões faciais que se torna e incômoda para quem o possui. De todos os artigos da literatura procurados nenhum apresenta um caso específico de aumento de coroa clínica inferior com objetivo estético.

\section{Conclusões}

O conhecimento das peculiaridades da biologia dos tecidos periodontais se torna primordial e eficaz para indicação de um tratamento pertinente e bem elaborado, o qual será obtido através de um diagnóstico clínico, condições e expectativas do paciente e habilidade técnica do cirurgião dentista.

Existem várias técnicas cirúrgicas para tratamento de sorriso gengival proveniente de Erupção Passiva Alterada. A cirurgia a retalho de espessura total com excisão de margens associada à osteotomia e osteoplastia da região anterior de mandíbula, relatada nesse caso, se mostrou eficaz e trouxe um retorno positivo por parte do paciente.

É escassa a literatura que estude mais sobre as dimensões e formas necessárias para uma estética harmoniosa também da região anteroinferior, que dê atenção a todos os elementos constituintes do sorriso, para gerar um resultado estético e satisfatório.

\section{Referências}

Alvarenga, D. B., Santana, C. L. V., Oliveira, F. R. D. T. S., Rodrigues, R. Q. F., Ribeiro, R. A. \& Sousa, J. N. L. (2018). Inter-relação periodontia/dentística na correção de sorriso gengival: relato de caso clínico. Braz J Periodontol. 28(2), 53-9.

Armitage, G. C. (1999). Developmentof a classification system for periodontal diseases and conditions. Ann Periodontol. 4(1), 1-6.

Bordin D., Nakanishi, F. A., Justo, F. R. M., \& Carnio J. (2010). Aumento de coroa clínica com objetivo estético. Revista PerioNews. 4(3), 225-31.

Braga, M. S., Nascimento, J. M. Q, Camargo, E. B., Veloso, F. J. M. S. C., Falcão, E.P., et al. (2014). Cirurgia plástica periodontal para correção de erupção passiva alterada. Braz J Periodontol. 24(4), 64-8.

Brilhante, F. V., Araújo, R. J. G., Mattos, J. L., Damasceno, J. M., Frota, L.V. \& Pinto, R. A. P. C. (2014). Cirurgia periodontal estética em dentes anteriores. Full Dent. Sci. 6(21), 39-44.

Dantas, A. A. R., Silva, E. R. C, \& Sako, J. S. (2012). Tratamento estético periodontal: revisão de literatura sobre alguns tipos de cirurgias. Rev. Odontol. Univ. Cid. São Paulo. 24(3), 226-34.

Flôres, M. M. D. Z., \& Tramontina, R. G. (1996). Aumento de coroa clínica: um conceito, uma realidade clínica ou uma verdade científica? RFO UPF. 1(1): $31-7$.

Gargiulo A. W., Wentz F. M., \& Orban B. (1961) Dimensions and relations of the dentogingival junction in human. J Periodont. $32(3)$, $261-7$.

Gonzales, M. K. S., \& Almeida, A. L. P. F. (2011). Erupção passiva alterada: diagnóstico, classificação e plano de tratamento. Revista Perionews. 5(1), 17-22.

Houais (2019). Dicionário Houaiss da Língua Portuguesa. Objetiva.

Kahn S., Menezes C. C, Tajra I, Dias A. T. \& Imperial R. C. (2014). Sorriso gengival: uma visão periodontal. Rev Clínica Ortodontia Dental Press. 13(4), 8896.

Lemes, L. T. O., Laufer, E., Reckziegel, M., Montenegro, M. M., \& Kampits, C. (2018). Aumento de coroa clínica com a técnica flapless: Relato de caso. Revista Peridontia. 28, 73-78.

Lindhe, J. \& Lang, N. P. (2018). Tratado de periodontia clínica e Implantodontia Oral. “In” Weijden, F. V. D., Slot, D. E., Echeverría, J. J., Lindhe, J. Controle Mecânico da Placa Supragengival. (6a ed.), Ed.Guanabara-Koogan. 36, 624-60.

Lindhe, J. \& Lang, N. P. (2018). Tratado de periodontia clínica e Implantodontia Oral. "In” Wennström, J. L. \& Lindhe J. Cirurgia Periodontal: Acesso cirúrgico. (6a ed.), Ed. Guanabara-Koogan. 36, 709-44. 
Research, Society and Development, v.10, n. 2, e26310212524, 2020

(CC BY 4.0) | ISSN 2525-3409 | DOI: http://dx.doi.org/10.33448/rsd-v10i2.12524

Lourenço, A. H. T., Júnior E. T. L., \& Silva, V. C. (2017). Aumento de coroa clínica- relato de caso. RFO. 22(3), 351-4.

Lukez, A., Pavlic, A., Trinajsticzrinski, M., \& Spalj, S. (2015). The unique contribution of elements of smile aesthetics to psychosocial well-being. J Oral Rehabil. 42(4), 275-81.

Mele, M., Felice, P., Sharma, P., Mazzotti, C., Bellone, P., \& Zucchelli, G. (2018). Esthetic treatment of altered passive eruption. Periodontology. 77(1), 65-83.

Pedron I. G. (2014). Associação terapêutica entre cirurgia gengival ressectiva e aplicação de toxina botulínica no sorriso gengival em paciente ortodôntico. Ortodontia SPO. 47(3), 245-9.

Pereira, A. S., Shitsuka, D. M., Parreira, F. J. \& Shitsuka, R. (2018). Metodologia da pesquisa científica. Ed. UAB/NTE/UFSM.

Pithon, M. M., Santos, A. M., Campos, M. S., Couto, F. S., Santos, A. F., Coqueiro, R. S., et al. (2014). Perceptionoflaypersonsand dental professionalsandstudents as regardstheaest hetic impactof gingival plasticsurgery. Eur J Orthod. 36(2), 173-8.

Tekavec, M. M., \& Tekavec, C. D. P. S. R. (1993). provides new patient management tool. Dent Econ. 83(4), 69-74.

Yin, R. K., (2015). O estudo de caso. Bookman. 